\title{
Fluorescence-Based Quantification of Gastrointestinal Perfusion: A Step Towards an Automated Approach
}

\author{
Harry G.M. Vaassen, BS, Bryan Wermelink, MS, ${ }^{1,2}$ \\ Robert H. Geelkerken, Prof., MD, PhD, ${ }^{1,2}$ and Daan J. Lips, MD, $\mathrm{PhD}^{3}$
}

\begin{abstract}
Background: Qualitative fluorescence angiography (FA) provides insights into intestinal tissue perfusion, but today it is not yet accurate in predicting anastomotic leakage. To improve peroperative detection of impaired perfusion, quantified parameters should be investigated using a standardized method. The aim of this study was to develop a (semi)automated algorithm for comprehensive and convenient analysis of FA parameters.

Materials and Methods: An analysis tool was developed for the extraction of quantified FA parameters. The start- and endpoint of intensity increase $\left(T_{0}\right.$ and $T_{\max }$ ) were automatically detected in the intensity-time curves. Algorithm performance was measured against manual assignment of $T_{0}$ and $T_{\max }$ by 9 independent observers in 18 in vivo generated test signals, using the intraclass correlation coefficient (ICC). Characteristics of parameter $T_{1 / 2}$ (time to $50 \%$ of maximal intensity) were analyzed in normally perfused small intestine of 32 subjects who underwent robotic laparoscopic surgery. Since ethical approval was not required under the Dutch law, the need for informed consent was waived.

Results: Automated detection of $T_{0}$ and $T_{\max }$ was successful in all subjects. Output of the algorithm had an excellent agreement with the median of the human observations: $\mathrm{ICC}=0.95$ (95\% confidence interval: $0.86-$ 0.96). Overall, $T_{1 / 2}$ had a median value of 5.1 (interquartile range $=2.4$ ) seconds and a minimal and maximal value of 1.3 and 9.9 seconds, respectively.

Conclusions: The presented method provided convenient data analysis in the search for effective FA quantification. Future research should expand the data to find adequate threshold values for peroperatively identifying insufficient perfusion and investigate the influence of physiological conditions.
\end{abstract}

Keywords: automated detection, fluorescence angiography, intestinal microcirculation, quantification

\section{Introduction}

I NADEQUATE LOCAL OR SYSTEMIC PERFUSION is regarded as a substantial factor in complications after gastrointestinal surgery. The most feared and frequent example is anastomotic leakage (AL), associated with strongly increased morbidity and mortality and incidence rates varying from $3 \%$ to $19 \% .^{1-4}$ In current practice, blood supply is evaluated peroperatively by means of clinical observations such as mucosal color, palpable pulsations, and bleeding from the marginal artery. However, these methods lack accuracy and objectivity, irrespective of a surgeon's experience. ${ }^{5}$ Alternative approaches including the use of Doppler, laser speckle contrast imaging, and hyperspectral imaging have yet failed to reach clinical implementa- tion, since no recommendation can be made regarding their applicability due to technical difficulties and low reproducibility. ${ }^{6-10}$ There is a need for objective and reliable peroperative detection of insufficient intestinal perfusion.

In recent years, fluorescence angiography (FA) has emerged as a promising tool for yielding intraoperative insight into local perfusion dynamics. FA involves the intravenous injection of contrast agent indocyanine green (ICG) and subsequent monitoring of its fluorescent appearance in bowel tissue. The role of FA in peroperative decision making during colorectal surgery has already been demonstrated. ${ }^{6,11-13}$ Furthermore, superiority of FA over judgment on clinical signs alone in the detection of perfusion impairment has been shown in experimental models. ${ }^{14,15}$ However, the acquired

\footnotetext{
${ }^{1}$ Multi-Modality Medical Imaging (M3i) Group, TechMed Centre, University of Twente, Enschede, The Netherlands.

${ }^{2}$ Section of Vascular Surgery, Department of Surgery, Medisch Spectrum Twente, Enschede, The Netherlands.

${ }^{3}$ Section of Gastrointestinal and Oncology Surgery, Department of Surgery, Medisch Spectrum Twente, Enschede, The Netherlands.
} 
images are evaluated in a qualitative manner, making them sensitive to misinterpretation. In addition, multicenter randomized controlled trials failed to show a significant reduction in AL after peroperative implementation of FA. ${ }^{12,13,16} \mathrm{It}$ becomes apparent that the current qualitative interpretation of FA is unreliable, creating the need for more objective quantification. ${ }^{16,17}$

Animal studies showed a great ability of quantified parameters to indicate blood flow and tissue ischemia. ${ }^{14,18}$ Quantification should be based on reproducible signal properties, meaning that circumstances other than tissue perfusion should not be influential. For this reason, the use of intensitybased parameters such as maximal intensity is not advised, since these depend on factors such as ICG dosing and the distance between tissue and camera. Time-based dynamics of the signal are more consistent. Parameter $T_{1 / 2}$, defined as the time between first intensity increase and the point of reaching $50 \%$ of the maximal intensity, showed promising predictive value for AL in colorectal surgery. ${ }^{7,19}$ However, as of yet there is no general consensus on the interpretation of quantified FA. ${ }^{16,20}$ Additional data obtained using a standardized method is required to distinguish normal and pathological findings.

This study presents a convenient and objective semiautomated method for the acquisition of peroperative quantitative parameters in FA. Moreover, as a first step toward a differentiating threshold value, the characteristics of the parameter $T_{1 / 2}$ in normally perfused small intestine are investigated.

\section{Materials and Methods}

\section{Data acquisition}

A total of 33 patients who underwent robotic laparoscopic abdominal surgery were prospectively included in this study. No ethical approval was needed under the Dutch law on medical research on human subjects, and the need for informed consent was, therefore, waived. All procedures were performed by 1 surgeon in Medisch Spectrum Twente (MST) Hospital (Enschede). No patient presented clinical signs suggesting impaired intestinal perfusion. Patient characteristics including laparoscopy indication are presented in Table 1. In each patient, FA was performed on the small intestine as a minor extension to standard peroperative FA application. No clinical decisions were made based on the intestinal FA analysis. All FA recordings were obtained using the Firefly module in the Da Vinci X system (Intuitive Surgical, Sunnyvale, CA, USA) after manual intravenous injection of $5 \mathrm{mg}$ ICG $(5 \mathrm{mg} / \mathrm{mL})$ and subsequent saline flushing.

\section{Parameter extraction}

A software tool for the quantitative analysis of FA recordings was developed in MATLAB R2019a (MathWorks, Natick, MA, USA). The main purpose of this tool was convenient quantification of FA with automated but usersupervised parameter extraction. The algorithm employed several steps. First, an intensity-time curve was determined by calculating the mean value of the green channel in a specific region of interest (ROI) for each timeframe. Signal noise was subsequently removed using a lowpass Butterworth filter (second order, cutoff frequency $=0.18 \mathrm{~Hz}$ ). The
Table 1. Characteristics of Patients Who UNDERWENT FLUORESCENCE ANGIOGRAPHY DURING Robotic Laparoscopic SURgery $(N=32)$

\begin{tabular}{lr}
\hline Characteristic & Data \\
\hline Age (years; mean \pm SD) & $66 \pm 12$ \\
Gender $(\%)$ & $19(59.4)$ \\
Female & $13(40.6)$ \\
Male & $25.7 \pm 5.3$ \\
BMI $\left(\mathrm{kg} / \mathrm{m}^{2}\right.$; mean $\left.\pm \mathrm{SD}\right)$ & \\
ASA score $(\%)$ & $4(12.5)$ \\
I & $20(62.5)$ \\
II & $8(25.0)$ \\
III & \\
Diagnosis $(\%)$ & $10(31.3)$ \\
Pancreatic malignancy & $6(18.8)$ \\
Colorectal liver metastases & $4(12.5)$ \\
Cholangiocarcinoma & $3(9.4)$ \\
Duodenum carcinoma & $3(9.4)$ \\
Cholecystolithiasis & $2(6.3)$ \\
Colon cancer & $4(12.5)$ \\
Other & \\
Vascular risk factors $(\%)$ & $7(24.1)$ \\
Smoking $(>5$ cigarettes per day) & $5(16.1)$ \\
Hypertension & $4(13.8)$ \\
Coronary disease & $3(9.4)$ \\
Diabetes & $2(6.7)$ \\
Vascular disease &
\end{tabular}

${ }^{\mathrm{a}}$ Includes intraductal papillary mucinous neoplasms.

ASA, American Society of Anaesthesia; BMI, body mass index; $\mathrm{SD}$, standard deviation.

following step was detection of the start- and endpoint of the intensity increase $\left(T_{0}\right.$ and $T_{\max }$; see Fig. 1$)$. For this purpose, the algorithm searched the intensity curve for points of change in linear trend. This comprised dividing the signal into segments in such a way that the following cost function is minimized:

$$
\begin{aligned}
& J(m, n)=\sum_{i=m}^{n-1}\left(x_{i}-\mu\left(\left[x_{m} \ldots x_{n-1}\right]\right)\right)^{2} \\
& -\frac{\left(\sum_{i=m}^{n-1}\left(x_{i}-\mu\left(\left[x_{m} \ldots x_{n-1}\right]\right)\right) \times(i-\mu([m \ldots n-1]))\right)^{2}}{\sum_{i=m}^{n-1}(i-\mu([m \ldots n-1]))^{2}}
\end{aligned}
$$

where $J(m, n)$ is the residual error, $x$ is the measured intensity in the ROI, and $m, n$ are index timeframes that divide the segments. After minimization of this expression by varying the segment division, the segment borders are considered change points in linear trend.

It is expected that the start- and endpoint of intensity increase are identified as one of these change points. Definitive designation of $T_{0}$ and $T_{\max }$ was performed using multiple conditions based on the slope of the signal. A more detailed description of the processing steps is provided in the Supplementary Data. $T_{1 / 2}$ was derived directly from $T_{0}$ and $T_{\max }$.

The performance of the algorithm in the detection of $T_{0}$ and $T_{\max }$ was evaluated by measuring its agreement with human observation. Nine independent observers appointed $T_{0}$ and $T_{\max }$ in a test data set of 18 signals. The test data set was generated by measuring the intensity in three random 


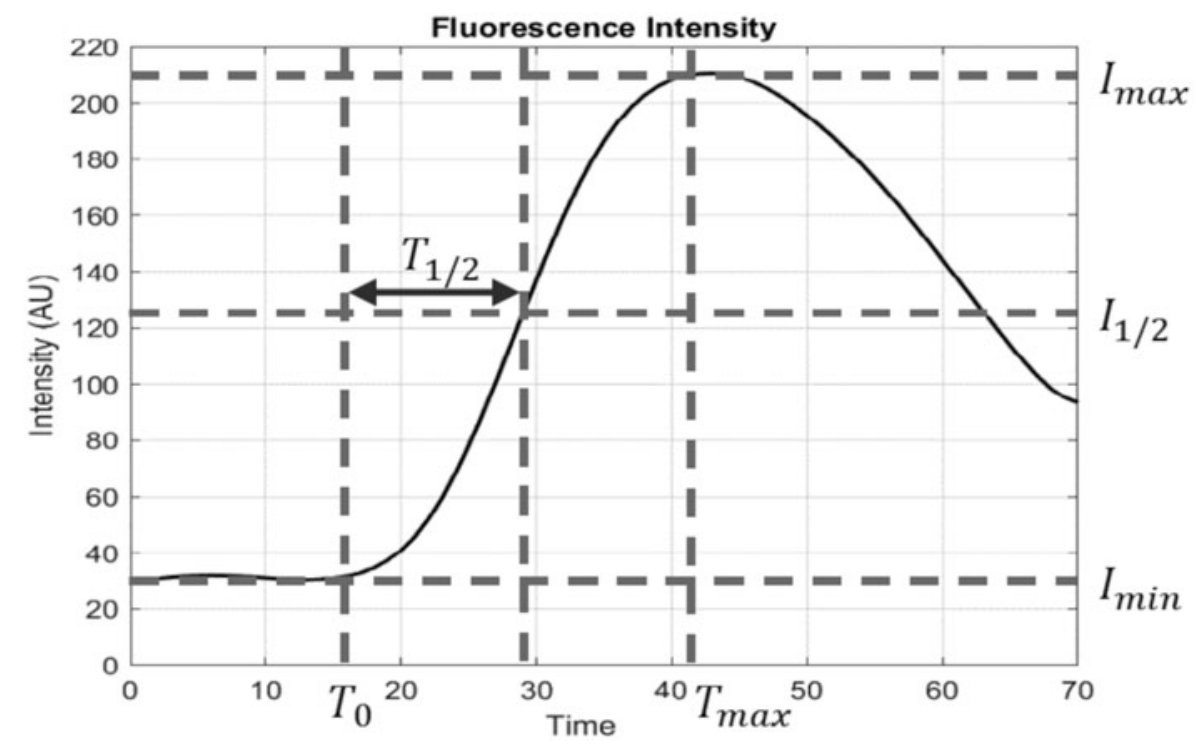

FIG. 1. Filtered intensity-time curve of FA. $T_{1 / 2}$ is defined as the time between first intensity increase and the point of reaching $50 \%$ of maximal intensity. FA, fluorescence angiography.

ROIs in the footage of 6 included patients. As measurement of agreement, the intraclass correlation coefficient (ICC) of the median human input and the algorithm output was computed using SPSS 26.0 (IBM Corp, Armonk, NY, USA). The intra- and interobserver variability was evaluated using the ICC as well.

\section{Patient analysis}

The characteristics of $T_{1 / 2}$ in patients without impaired perfusion were analyzed in a semiautomated manner. In each recording, the small intestine was manually segmented. The segmentation was subsequently divided into smaller polygons according to a $10 \times 10$ grid (creating multiple ROIs), whereupon $T_{1 / 2}$ was calculated automatically for each polygon. A manifold of measurements in each recording allowed for the assessment of patient-specific variations in $T_{1 / 2}$. The characteristics of $T_{1 / 2}$ were investigated using the fivenumber summary (i.e., median, minimum, maximum, and upper/lower quarters) of all measurements in each patient. Data points were considered outliers when they exceeded the 75th percentile plus 1.5 times the interquartile range (IQR) or were inferior to the 25 th percentile minus 1.5 times the IQR. In addition, by assigning a color to each polygon based on the value of $T_{1 / 2}$, a cartogram was developed for each patient.

\section{Results}

FA was performed on a total of 33 patients, who all had a prosperous postoperative recovery. The recording of 1 patient was excluded from the analysis of $T_{1 / 2}$ characteristics, due to faulty ICG admission.

\section{Algorithm performance}

Among the 9 observers, ICC varied between 0.93 and 0.99 (95\% confidence interval [CI]: $0.89-0.99$ ) for $T_{0}$. For $T_{\max }$, intraobserver ICC varied between 0.96 and 1.00 (95\% CI: 0.91-1.00). The ICC across all observers was 0.95 (95\% CI:
0.92-0.98) for $T_{0}$ and 0.98 (95\% CI: 0.96-0.99) for $T_{\max }$. Comparison of the algorithm with the median of the human observations provided an ICC of 0.95 (95\% CI: 0.86-0.96).

\section{Patient analysis}

A total of 32 patients were analyzed using the developed algorithm. In Figure 2, box plots are displayed that indicate the characteristics of $T_{1 / 2}$ in the small intestine for all included patients. The median value of $T_{1 / 2}$ varied between 2.1 and 9.1 seconds and the IQR varied between 0.3 and 2.9 seconds. Overall, $T_{1 / 2}$ had a median value of $5.1(\mathrm{IQR}=2.4)$ seconds and a minimal and maximal value of 1.3 and 9.9 seconds, respectively. The mean $T_{1 / 2}$ was found to be 5.2 $(\sigma=1.9)$ seconds, with 95\% CI: $1.5-9.0$.

Figure 3 shows two examples of a cartogram automatically generated based on the value of $T_{1 / 2}$, as well as the grid-based division of an intestinal segmentation.

\section{Discussion}

This pilot study demonstrated that an automated approach in the quantification of FA for peroperative intestinal perfusion assessment including the characteristics of FA parameter $T_{1 / 2}$ is feasible.

The ability of the change point detection algorithm was examined. Since there is no common consensus on the exact definition of $T_{0}$ and $T_{\max }$, an inquiry on the input of 9 independent human observers was performed. With intraobserver ICCs varying between 0.93 and 1.000 , it was found that these observers allocated $T_{0}$ and $T_{\max }$ with excellent consistency. Moreover, the interobserver ICC of 0.95 showed a great agreement between observers. This substantiated the decision to consider the input of multiple human observers as "golden standard" in the evaluation of algorithm performance. The time-efficient change point detection algorithm showed excellent agreement with the observers $(\mathrm{ICC}=0.95)$. This was considered as proper validation, advocating the use of an unsupervised method (applying the grid) in the 


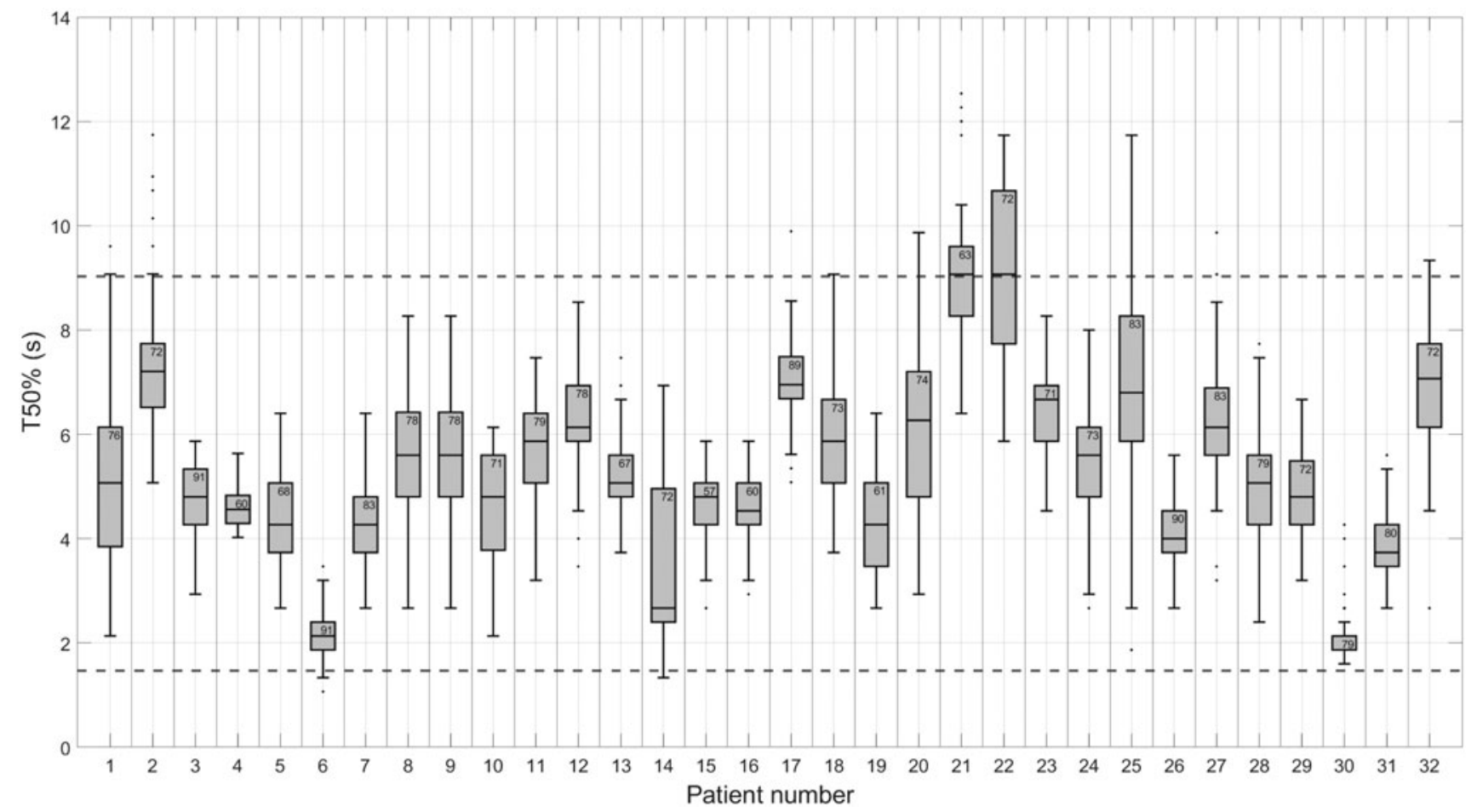

FIG. 2. Box plots representing the measurements of $T_{1 / 2}$ in the small intestine for all included patients. The total number of ROIs measured in each patient is displayed inside the box plots. Small dots represent measurements in ROIs that were considered outliers. Dotted lines represent $95 \%$ CI for $T_{1 / 2}$. CI, confidence interval; ROI, region of interest.

analysis of $T_{1 / 2}$. The benefits of the semiautomated algorithm are best illustrated with the presented cartograms. Immediate perfusion quantification can be achieved in large sections of the intestine with one injection of ICG.

Automated analysis of $T_{1 / 2}$ was performed in FA recordings of 32 normally perfused small intestines. The found mean value of $5.2(\sigma=1.9)$ seconds is considered to be fast, compared with results reported by Wada et al. ${ }^{7}$ and Son et al. ${ }^{19}$ In these studies, colonic perfusion in patients who underwent surgery for colorectal cancer was quantitatively analyzed using FA. Their presented mean values of $T_{1 / 2}$ were, respectively, $12.5(\sigma=7.6)$ and $11.7(\sigma=0.8)$ seconds for patients who did not develop AL. Based on the results of this pilot study, it is expected that $T_{1 / 2}$ values are generally lower in the small intestine than in the large intestine. Son et al. showed a significant increase of $T_{1 / 2}$ in patients who presented AL, reporting a mean of $40.37(\sigma=7.8)$ seconds. Despite the number of patients with $\operatorname{AL}(n=6)$ being relatively small, these results are promising for the establishment of a useful threshold value. This study reveals a spread of parameter $T_{1 / 2}$ between 1.5 and 9.0 seconds in normally perfused small intestine. When in future research patients with impaired intestinal perfusion show values relatable to the AL cases presented by Son et al., a great predictive value could be achieved.

It should be considered that the single parameter $T_{1 / 2}$ is unlikely to portray the complete perfusion situation in a tissue area. Several circumstances can be imagined where impaired perfusion is not indicated by $T_{1 / 2}$. As described by Barberio et al., bowel tissue with extreme perfusion impairment might show a "normal" value of a time-based parameter such as $T_{1 / 2}{ }^{21}$ When the intensity levels substantially decrease, a time-to-intensity threshold becomes severely inaccurate because the signal-to-noise ratio decreases. In this case, a lot of information about the signal increase is lost, weakening the capability of time-based parameters. However, in the described situation of severely impaired transmural perfusion, the regular clinical judgment improves its informative value: black discolored bowel tissue is not suitable for anastomosis, and it is insensible to perform FA in this situation. The value of quantified FA lies in marginal perfusion. ${ }^{21}$ It is, however, unclear where the strength of clinical judgment stops and the benefit of FA begins.

To clarify the correct interpretation of quantified FA and be able to identify inadequate perfusion, this study has to be expanded with more data. The presented approach is a substantial step toward an objective standardized method of analysis. When this is established, large scale data acquisition should be relatively straightforward. Naturally, the succeeding step would be to include patients with a known form of perfusion impairment. For instance, patients suffering from acute mesenteric ischemia (AMI) or chronic mesenteric ischemia (CMI) are certainly relevant. Indeed, one of the most important applications of FA is in the advanced stages of AMI or CMI. ${ }^{22,23}$ In case of transmural ischemia, bowel tissue is often surgically removed. It is very likely that the surrounding non-necrotic tissue is not properly perfused in this situation, increasing the risk of AL. A predictive threshold value in combination with the presented cartograms could be of great assistance in these circumstances.

A limitation in the current method is the lack of standardized admission of ICG. The rate and anatomical site at which the agent is injected influence the dilution in blood. This consequently affects intensity levels and fluorescence 


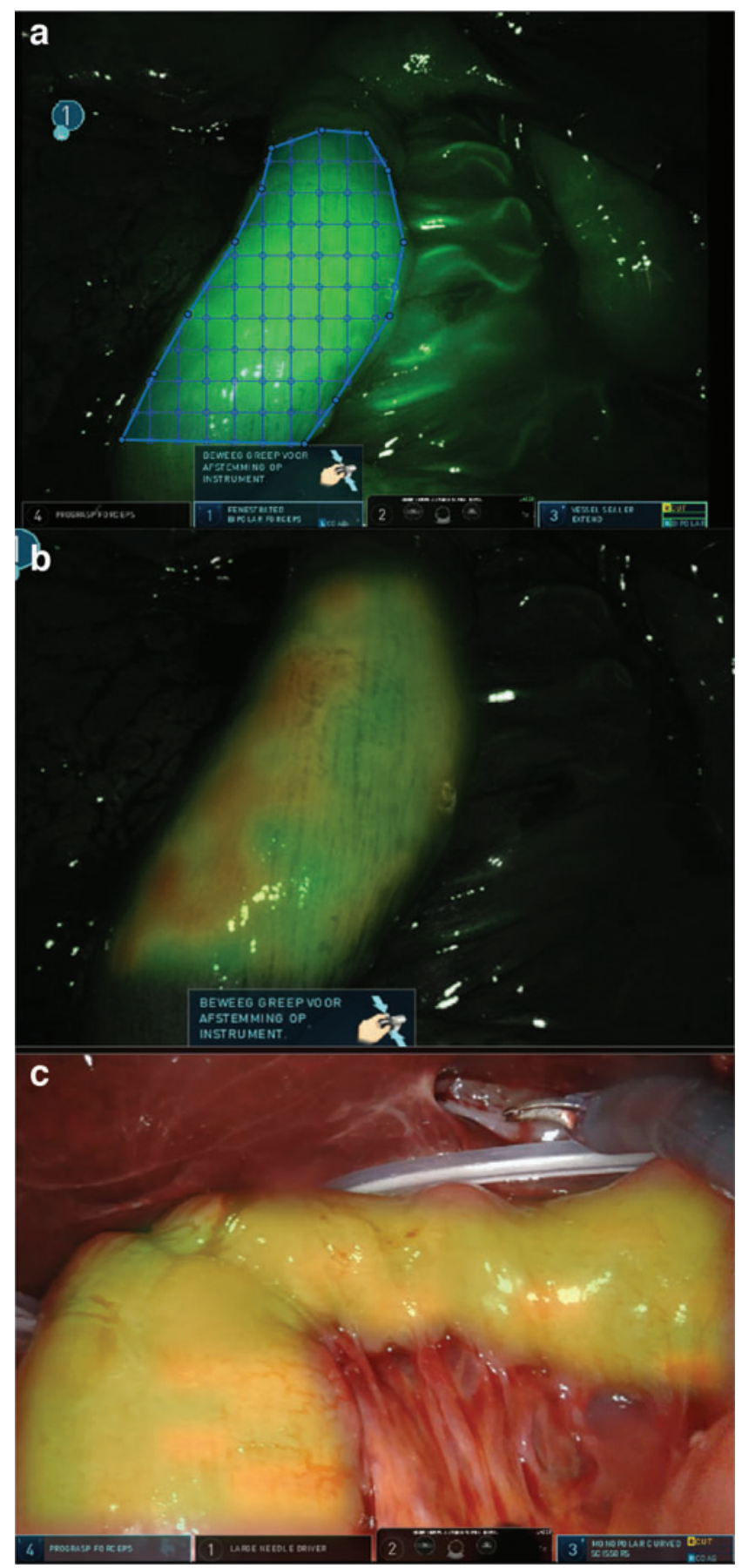

FIG. 3. (a) Manual segmentation and partitioning of small intestine in FA recording. (b) Example of cartography based on $T_{1 / 2}$, displayed over the view of the FA camera before ICG injection. The color range is based on the range of $T_{1 / 2}$ in each patient. (c) Example of a cartogram merged into the regular white light image. FA, fluorescence angiography; ICG, indocyanine green. Color images are available online.

dynamics. It is, therefore, advised to investigate the use of an automated contrast injection system. Another presumably influential factor in the dynamics of ICG is cardiac output. In case of high cardiac output, it can be expected that time-based perfusion parameters improve. Future study should investigate the effect of cardiac output on quantified FA parameters and consider a possible correction. Finally, it is advised to examine the effects of temperature on FA parameters. Owing to consistent carbon dioxide insufflation, the temperatures during laparoscopy are expected to be similar. However, these conditions change when FA is applied in open procedures.

\section{Conclusion}

In conclusion, the presented semiautomated approach allows convenient analysis of FA recordings and can be of great assistance in the pursuit of threshold values to distinguish peroperative sufficient and impaired bowel wall perfusion. Clinical validation studies should be undertaken to underline that semiautomated FA analysis could reliably identify nonviable bowel tissue in gastrointestinal surgery.

\section{Authors' Contributions}

H.G.M.V., B.W., R.H.G., and D.J.L. designed the study. D.J.L. acquired data. H.G.M.V., B.W., R.H.G., and D.J.L. analyzed the data. All authors participated in data interpretation and article preparation.

\section{Disclosure Statement}

No competing financial interests exist.

\section{Funding Information}

No funding was received.

\section{Supplementary Material}

Supplementary Data

\section{References}

1. Platell C, Barwood N, Dorfmann G, Makin G. The incidence of anastomotic leaks in patients undergoing colorectal surgery. Color Dis 2007;9:71-79.

2. Kingham TP, Pachter HL. Colonic anastomotic leak: Risk factors, diagnosis, and treatment. J Am Coll Surg 2009;208: 269-278.

3. Turrentine FE, Denlinger CE, Simpson VB, et al. Morbidity, mortality, cost, and survival estimates of gastrointestinal anastomotic leaks. J Am Coll Surg 2015;220:195206.

4. Urbanavičius L. How to assess intestinal viability during surgery: A review of techniques. World J Gastrointest Surg 2011;3:59.

5. Karliczek A, Harlaar NJ, Zeebregts CJ, Wiggers T, Baas PC, van Dam GM. Surgeons lack predictive accuracy for anastomotic leakage in gastrointestinal surgery. Int $\mathbf{J}$ Colorectal Dis 2009;24:569-576.

6. Kawada K, Hasegawa S, Wada T, et al. Evaluation of intestinal perfusion by ICG fluorescence imaging in laparoscopic colorectal surgery with DST anastomosis. Surg Endosc 2017;31:1061-1069.

7. Wada T, Kawada K, Takahashi R, et al. ICG fluorescence imaging for quantitative evaluation of colonic perfusion in laparoscopic colorectal surgery. Surg Endosc 2017;31: 4184-4193.

8. Kim JC, Lee JL, Park SH. Interpretative guidelines and possible indications for indocyanine green fluorescence 
imaging in robot-assisted sphincter-saving operations. Dis Colon Rectum 2017;60:376-384.

9. Rønn JH, Nerup N, Strandby RB, et al. Laser speckle contrast imaging and quantitative fluorescence angiography for perfusion assessment. Langenbeck's Arch Surg 2019; 404:505-515.

10. Björck M, Koelemay M, Acosta S, et al. Editor's choiceManagement of the diseases of mesenteric arteries and veins clinical practice guidelines of the European Society of Vascular Surgery (ESVS). Eur J Vasc Endovasc Surg 2017;53:460-510.

11. $\mathrm{Su} \mathrm{H}, \mathrm{Wu} \mathrm{H}, \mathrm{Bao} \mathrm{M}$, et al. Indocyanine green fluorescence imaging to assess bowel perfusion during totally laparoscopic surgery for colon cancer. BMC Surg 2020;20: $1-7$.

12. De Nardi P, Elmore U, Maggi G, et al. Intraoperative angiography with indocyanine green to assess anastomosis perfusion in patients undergoing laparoscopic colorectal resection: Results of a multicenter randomized controlled trial. Surg Endosc 2020;34:53-60.

13. Jafari MD, Wexner SD, Martz JE, et al. Perfusion assessment in laparoscopic left-sided/anterior resection (PILLAR II): A multi-institutional study. J Am Coll Surg 2015;220: 82-92.e1.

14. Diana M, Noll E, Diemunsch P, et al. Enhanced-reality video fluorescence: A real-time assessment of intestinal viability. Ann Surg 2014;259:700-707.

15. Matsui A, Winer JH, Laurence RG, Frangioni JV. Predicting the survival of experimental ischaemic small bowel using intraoperative near-infrared fluorescence angiography. Br J Surg 2011;98:1725-1734.

16. Lütken CD, Achiam MP, Svendsen MB, Boni L, Nerup N. Optimizing quantitative fluorescence angiography for visceral perfusion assessment. Surg Endosc 2020;34:52235233.

17. Degett TH, Andersen HS, Gögenur I. Indocyanine green fluorescence angiography for intraoperative assessment of gastrointestinal anastomotic perfusion: A systematic review of clinical trials. Langenbeck's Arch Surg 2016;401:767-775.

18. Nerup N, Andersen HS, Ambrus R, et al. Quantification of fluorescence angiography in a porcine model. Langenbeck's Arch Surg 2017;402:655-662.

19. Son GM, Kwon MS, Kim Y, Kim J, Kim SH, Lee JW. Quantitative analysis of colon perfusion pattern using indocyanine green (ICG) angiography in laparoscopic colorectal surgery. Surg Endosc 2019;33:1640-1649.

20. Vallance A, Wexner S, Berho M, et al. A collaborative review of the current concepts and challenges of anastomotic leaks in colorectal surgery. Color Dis 2017;19:O1O12.

21. Barberio M, Felli E, Seyller E, et al. Quantitative fluorescence angiography versus hyperspectral imaging to assess bowel ischemia: A comparative study in enhanced reality. Surgery 2020;168:178-184.

22. Karampinis I, Keese M, Jakob J, et al. Indocyanine green tissue angiography can reduce extended bowel resections in acute mesenteric ischemia. J Gastrointest Surg 2018;22: 2117-2124.

23. Nakagawa Y, Kobayashi K, Kuwabara S, Shibuya H, Nishimaki T. Use of indocyanine green fluorescence imaging to determine the area of bowel resection in nonocclusive mesenteric ischemia: A case report. Int J Surg Case Rep 2018;51:352-357.

Address correspondence to:
Harry G.M. Vaassen, BS
Multimodality Medical Imaging (M3i) Group
Techmed Centre
University of Twente
Drienerlolaan 5
Enschede $7522 \mathrm{NB}$
The Netherlands
E-mail: harry.vaassen@gmail.com

Address correspondence to: ing (M3i) Group versity of Twente Drienerlolaan 5 schede $7522 \mathrm{NB}$ E-mail: harry.vaassen@gmail.com 